\title{
Profilo lipidico e terapia antiretrovirale in una coorte di bambini, adolescenti e giovani adulti con infezione da HIV a trasmissione verticale. Lipid profile and antiretroviral therapy in a cohort of children, adolescents and young adults infected with HIV by vertical transmission.
}

\author{
Laura Bisoffi, Daniele Donà, Carlo Giaquinto, Osvalda Rampon
}

Dipartimento per la Salute della Donna e del Bambino, Università degli Studi di Padova, Azienda Ospedaliera

\section{Autore}

per la corrispondenza:

\section{Osvalda Rampon}

Clinica Pediatrica, Azienda

Ospedaliera di Padova,

Via Giustiniani, 3

35128 Padova, Italy

osvalda.rampon@aopd.veneto.it

\section{Keywords:}

Children, young adults, HIV combined antiretroviral therapy, protease inhibitor, body mass index, HDL, LDL

\section{Potenziali conflitti}

di interesse: nessuno

JHA 2017; 2(3): 65-70

DOI: 10.19198/JHA31435

\section{Riassunto}

Con l'introduzione della terapia antiretrovirale combinata (CART), l'infezione da HIV è diventata una patologia cronica, associata ad aterosclerosi accelerata e aumentato rischio di eventi cardiovascolari precoci. Numerosi studi, svolti sia nella popolazione adulta sia in quella pediatrica, hanno identificato l'origine multifattoriale di questo aumentato rischio cardiovascolare: l'effetto diretto del virus, l'infiammazione cronica e la prolungata esposizione alla terapia antiretrovirale concorrono infatti, insieme ai fattori di rischio classici, a determinare un'alterazione del profilo lipidico in senso aterosclerotico.

II nostro lavoro è uno studio osservazionale descrittivo trasversale, condotto su 76 pazienti seguiti presso l'Ambulatorio di Malattie infettive pediatriche dell'Azienda Ospedaliera di Padova. La popolazione comprende 35 maschi e 41 femmine, con range d'età 6-34 anni ed età media 21.1 anni; tutti i pazienti sono affetti da infezione da HIV acquisita per via verticale e tutti eccetto uno sono in CART. II nostro scopo principale è stato indagare la prevalenza di alterazioni del profilo lipidico e la loro relazione con il tipo di terapia assunta e con lo stato viro-immunologico e infiammatorio. Lo studio ha evidenziato una quota elevata (57.9\%) di pazienti con uno o più parametri lipidici alterati tra colesterolo totale, LDL, HDL e trigliceridi; per quanto riguarda la relazione tra alterazioni del profilo lipidico e terapia antiretrovirale, non abbiamo però rilevato associazioni significative. Quella dei pazienti pediatrici e giovani adulti con infezione verticale da HIV è quindi una popolazione ad elevato rischio di aterosclerosi precoce ed eventi cardiovascolari; la prevenzione è perciò fondamentale, sia a livello primario, sia a livello $d i$ screening e diagnosi precoce della patologia cardiovascolare.

\section{Abstract}

Following highly active antiretroviral therapy (HAART) introduction, HIV infection has become a chronic disease, characterized by premature atherosclerosis and increased incidence of cardiovascular events. Many studies, conducted both on adults and children, have shown that multiple factors are associated with this increased cardiovascular risk; direct viral effect, chronic inflammation, long lasting antiretroviral exposition and general cardiovascular risk factors contribute to the developement of dyslipidemia and consequently to increased cardiovascular risk.

Our cross-sectional study has been conducted on $76 \mathrm{pa-}$ tients followed at the outpatient clinic for pediatric infectious diseases of Padua Hospital. The population included 35 boys and 41 girls, aged 6-34 years old (mean age 21.1 years); all patients have a perinatally-acquired HIV infection and 75 of them are on HAART. The primary aim of this study was to investigate the prevalence of lipid abnormalities and their relation with HAART regimen and with biomarkers of viro-immunological state and inflammation. We found a high prevalence of dyslipidemia among our patients (57.9\%), with increased total cholesterol, LDL-cholesterol and triglycerides and low LDL-cholesterol. We did not find any significant difference in frequency of lipid profile alterations between patients treated with HAART regimens including protease inhibitors and patients treated with other regimes.

Children and young adults with vertically-acquired HIV infection are exposed to premature atherosclerosis and cardiovascular events risk. Prevention is therefore very important, both by education and screening for early diagnosis of cardiovascular disease.

\section{Introduzione}

Come per gli adulti, la terapia antiretrovirale combinata (CART) ha drammaticamente ridotto la morbilità e la mortalità tra i bambini con infezione da HIV acquisita per via verticale, che è quindi diventata una malattia cronica dell'infanzia. 
Sebbene molti dei bambini che si sono infettati nei primi anni dell'epidemia siano ormai deceduti, un numero sempre crescente di pazienti infettatisi in epoca perinatale sopravvive fino all'adolescenza e all'età adulta (1). Questo crea ovviamente nuove sfide, soprattutto quella del controllo degli effetti collaterali a lungo termine dei farmaci, ma anche degli effetti cronici dell'infezione sui diversi organi e apparati.

Con la riduzione della mortalità per infezioni opportunistiche e neoplasie AIDS-definenti, sono emerse nuove problematiche per i pazienti con infezione da HIV, con la patologia cardiovascolare che è diventata la prima causa di morbilità e mortalità (2). L'aumentato rischio cardiovascolare di questi pazienti deriva dall'interazione tra fattori di rischio classici (età, sesso, familiarità, dislipidemia, ipertensione arteriosa, diabete, fumo, inattività fisica e sovrappeso/obesità) (3) e fattori di rischio specifici, direttamente legati all'infezione da HIV (4).

Questi ultimi comprendono:

1) la capacità del virus sul metabolismo lipidico, che I'HIV è in grado di modificare direttamente; nei pazienti adulti sia un'elevata viremia che una bassa conta dei CD4 correlano con bassi livelli di lipoproteine ad alta densità (HDL) (5);

2) gli effetti collaterali dei farmaci: il profilo lipidico tipico dei pazienti in CART, soprattutto con inibitori delle proteasi $(\mathrm{PI})$, è caratterizzato da aumento dei trigliceridi e del colesterolo totale e riduzione delle $\mathrm{HDL}$, con o senza aumento delle lipoproteine a bassa densità (LDL); anche altri effetti collaterali come lo sviluppo di insulino-resistenza e diabete e la lipodistrofia possono incidere sul rischio cardiovascolare; inoltre la terapia avrebbe un effetto negativo anche sulla pressione arteriosa $(6,7)$;

3) lo stato di prolungata immunoattivazione conseguente alla cronicizzazione dell'infezione, con conseguente infiammazione cronica che contribuisce all'alterazione del profilo lipidico e porta ad un' aterogenesi accelerata $(8,9)$.

I fattori di rischio classici sono condizioni estremamente frequenti, cui la popolazione HIV è sottoposta come la popolazione generale; diventa pertanto difficile distinguere quale quota del rischio cardiovascolare sia da attribuire ai fattori classici e quale ai fattori specifici, perché vari possono essere gli elementi confondenti.

Nonostante non siano presenti in letteratura dati sull'incidenza di eventi cardiovascolari nei bambi- ni con infezione da HIV, è evidente come essi siano una popolazione a rischio, data l'aumentata incidenza di aterosclerosi precoce subclinica e di alterazioni del profilo lipidico, documentata in diversi studi; questo va sommato al fatto che essi, rispetto alla popolazione di pazienti infettati in età adulta, subiscono un'esposizione più prolungata sia all'infezione sia alla terapia. In una coorte di pazienti tra i 4 e i 19 anni con infezione da HIV, la prevalenza di ipercolesterolemia è stata calcolata al $13 \%$, contro il $4.8 \%$ rilevato nella coorte non infetta di pari età; i fattori correlati con maggior forza all'ipercolesterolemia erano l'assunzione di PI e l'età tra i 4 e i 6 anni (10). Un altro lavoro ha analizzato la prevalenza di ipercolesterolemia e di ipertrigliceridemia in una popolazione di 178 bambini infetti, ripetendo periodicamente gli esami ematochimici nell'arco di 5 anni; l'età media dei pazienti al primo prelievo era di 6.3 anni, l'81\% era in terapia antiretrovirale e il 56\% assumeva un PI. I $47 \%$ dei bambini ha avuto almeno un prelievo indicante ipercolesterolemia e il $67 \%$ almeno uno indicante ipertrigliceridemia. L'assunzione di PI, la viremia non rilevabile e l'appartenenza alla categoria immunologica 3 del CDC sono stati evidenziati come fattori associati a maggior incidenza di ipercolesterolemia, mentre l'assunzione di PI e un body mass index (BMI) $>90^{\circ}$ percentile per sesso ed età erano associati allo sviluppo di ipertrigliceridemia. Ancora una volta, quindi, I'assunzione di PI sembra essere un fattore determinante nella patogenesi dell'alterazione lipidica (11). Un altro studio si è focalizzato su questo aspetto, confrontando la prevalenza delle alterazioni del profilo lipidico, all'interno di una popolazione di 37 pazienti con infezione da HIV in terapia antiretrovirale e range d'età 1-17 anni, tra i pazienti trattati con regimi contenenti $\mathrm{PI}$ e quelli invece trattaticon regimi PI-free. Per il gruppo esposto ai PI sono stati evidenziati livelli più elevati di colesterolo totale, LDL e trigliceridi, mentre non sono state riscontrate differenze per quanto riguarda le HDL (12).

\section{Materiali e metodi}

Gli obiettivi del nostro studio osservazionale trasversale condotto su una popolazione di pazienti pediatrici e giovani adulti con infezione da HIV verticale che hanno avuto accesso all'Ambulatorio di Malattie infettive del Dipartimento per la Salute della Donna e del Bambino di Padova nel periodo 


\begin{tabular}{|c|c|}
\hline Caratteristiche della popolazione & $\mathbf{N}(\%)$ o media (ds) \\
Totale pazienti & $76(100 \%)$ \\
Femmine & $41(53.9 \%)$ \\
Maschi & $35(46.1 \%)$ \\
Caucasici & $50(65.8 \%)$ \\
Africani & $24(31.6 \%)$ \\
Altre etnie & $2(2.6 \%)$ \\
Età (anni) & $21.1(6.6)$ \\
Co-infezione HBV e/o HCV & $9(11.8 \%)$ \\
Fumo & $29(38.2 \%)$ \\
In terapia HAART & $75(98.7 \%)$ \\
\hline
\end{tabular}

Tabella 1. Caratteristiche generali della popolazione in studio.

\begin{tabular}{c|c}
\hline $\begin{array}{c}\text { Caratteristiche } \\
\text { viro-immunologiche e terapia }\end{array}$ & $\begin{array}{c}\mathbf{N}(\%) \\
\text { o media (ds) } \\
\text { CD4 totali (cellule/microL) }\end{array}$ \\
$714(277)$ \\
CD4 percentuali sul totale dei linfociti (\%) & $31.5(9.5)$ \\
Rapporto CD4/CD8 & $0.99(0.52)$ \\
Carica virale < 34 copie/ml & $62(81.6 \%)$ \\
In terapia HAART & $75(98.7 \%)$ \\
In terapia con regimi contenenti inibitori delle proteasi & $52(68.4 \%)$ \\
Pazienti che non assumono inibitori di proteasi & $24(31.6 \%)$ \\
(compreso il paziente non in terapia)
\end{tabular}

Tabella 2. Caratteristiche della popolazione relative alla terapia e allo stato viro-immunologico.

\begin{tabular}{|c|c|}
\hline $\begin{array}{c}\text { Caratteristiche relative ai fattori di rischio } \\
\text { cardiovascolare classici }\end{array}$ & $\begin{array}{c}\mathbf{N}(\%) \\
\text { o media (ds) }\end{array}$ \\
BMI (kg/m2) & $21.70(3.59)$ \\
Fumo & $29(38.2 \%)$ \\
Pressione Sistolica $(\mathrm{mmHg})$ & $117.3(13.7)$ \\
Pressione Diastolica $(\mathrm{mmHg})$ & $71.2(10.5)$ \\
Glicemia a digiuno $(\mathrm{mmol} / \mathrm{L})$ & $4.63(0.74)$ \\
\hline
\end{tabular}

Tabella 3. Caratteristiche della popolazione relative ai fattori di rischio cardiovascolari classici.
3) lipodistrofia e la sua relazione con il tipo di terapia assunta e le alterazioni del profilo lipidico,

4) fattori di rischio cardiovascolari classici, come abitudine al fumo, ipertensione arteriosa e sovrappeso. Sono stati inclusi 76 pazienti con infezione verticale da HIV, di età compresa tra 6 e 34 anni, le cui caratteristiche sono riportate nella Tabella 1.

Per ciascun paziente sono stati raccolti dati clinico-anamnestici (età, sesso, etnia, stadiazione della malattia, peso, altezza e BMI, pressione arteriosa, lipodistrofia, abitudine al fumo, coinfezioni, regime terapeutico) e dati laboratoristici (CD4 assoluti e percentuali, CD8\%, rapporto $\mathrm{CD} 4 / \mathrm{CD} 8$, carica virale, glicemia, colesterolo totale, colesterolo-LDL, colesterolo-HDL e trigliceridi ( $\mathrm{mmol} / \mathrm{L}$ ) a digiuno, Apolipoproteina B, D-dimero, IL-6. Per i parametri del profilo lipidico, sono stati utilizzati i range di normalità degli adulti, per rendere lo studio confrontabile con la letteratura (ad esempio nello studio di Carter et al. (11), svolto su una popolazione pediatrica, vengono applicati i valori lipidici desiderabili per gli adulti).

\section{Risultati}

Lo stato dei nostri pazienti relativamente a condizione viro-immunologica, tipo di terapia e altri fattori di rischio cardiovascolare è rappresentato nelle Tabelle 2 e 3.

Una quota elevata di pazienti (57.9\%) ha uno o più parametri lipidici al di fuori dei valori desiderabili; considerando l'intera popolazione, quello più frequentemente alterato è il colesterolo-HDL, inferiore ai valori desiderabili nel $36.8 \%$ della popolazione. È stata riscontrata ipercolesterolemia nel $18.4 \%$ dei pazienti, prevalenza superiore al 13\% evidenziato nello studio di Farley et al. (10), svolto però su una popolazione più giovane. La frequenza di alterazione è significativamente più elevata nelle femmine rispetto ai maschi per quanto riguarda colesterolo totale e LDL; lo stesso vale per il confronto tra i valori medi dei diversi parametri. Il colesterolo-HDL e i trigliceridi risultano invece alterati con una frequenza sovrapponibile tra i due sessi Figura 1. Nel confronto invece tra i soggetti pediatrici (età $\leq 18$ anni) e i giovani adulti, I'unico parametro la cui frequenza di alterazione mostra una differenza statisticamente significativa è il colesterolo-HDL, inferiore ai valori desiderabili nel 53.3\% dei pazienti fino a 18 anni, contro il $26.1 \%$ dei pazienti adulti; per quanto riguarda gli altri parametri, la differenza di frequenza tra i

gennaio-maggio 2017, sono stati quelli di valutare la prevalenza di:

1) alterazioni del profilo lipidico e la loro eventuale correlazione con la condizione viroimmunologica e il tipo di cART,

2) valori elevati di alcuni parametri infiammatori e la loro correlazione con le alterazioni dei lipidi, 


\section{articolo originale}

due gruppi è molto meno marcata e non statisticamente significativa. Considerando i valori medi, la differenza tra i due gruppi è invece statisticamente significativa per tre parametri: nei pazienti adulti si riscontrano livelli medi di HDL inferiori e livelli medi di trigliceridi e Apolipoproteina B superiori rispetto ai pazienti pediatrici, fattori che determinano quindi, nel complesso, un rischio cardiovascolare più elevato.

Suddividendo la popolazione in base al tipo di regime antiretrovirale assunto, abbiamo rilevato una frequenza di alterazione superiore nel gruppo che non assume PI per quanto riguarda colesterolo totale e HDL, mentre la frequenza di alterazione si è rivelata superiore nei pazienti in terapia con PI per quanto riguarda LDL e trigliceridi Figura 2. Nessuna di queste differenze è però statisticamente significativa dopo l'applicazione del Test chi-quadro. Anche considerando i valori medi dei vari parametri lipidici la differenza tra il gruppo esposto ai $\mathrm{Pl}$ e quello non esposto non è statisticamente significativa. I nostri risultati differiscono quindi da quanto riportato in letteratura per $\mathrm{i}$ pazienti pediatrici $(10,11)$, dove i PI si associano più frequentemente delle altre classi di farmaci antiretrovirali ad alterazioni del profilo lipidico. La mancata significatività dell'associazione tra trattamento con $\mathrm{Pl}$ e alterazioni lipidiche rilevata nel nostro studio potrebbe essere in parte spiegata dalla scarsa numerosità del campione. Inoltre, i due studi sopra citati risalgono al rispettivamente al 2005 e al 2006; negli ultimi anni sono stati apportati vari miglioramenti ai $\mathrm{PI}$, con riduzione degli effetti avversi ad essi associati, ed è quindi possibile che questa sia una delle ragioni della non significatività delle differenze tra chi li assume e chi no. Per quanto riguarda l'associazione tra lipodistrofia e profilo lipidico, non è stata evidenziata per alcun parametro del profilo lipidico una frequenza di alterazione superiore nei pazienti con diagnosi clinica di lipodistrofia rispetto a quelli non affetti da questa condizione. Questo potrebbe essere dovuto, almeno in parte, alla ridotta numerosità della nostra popolazione; in particolare la lipodistrofia è relativamente poco frequente (15 pazienti, pari al 19.7\% della popolazione), mentre l'alterazione di uno o più parametri lipidici è molto più frequente (44 pazienti, il 57.9\% del totale). Questo risultato conferma l'ipotesi che il danno metabolico e morfologico abbiano eziologia multifattoriale (13).

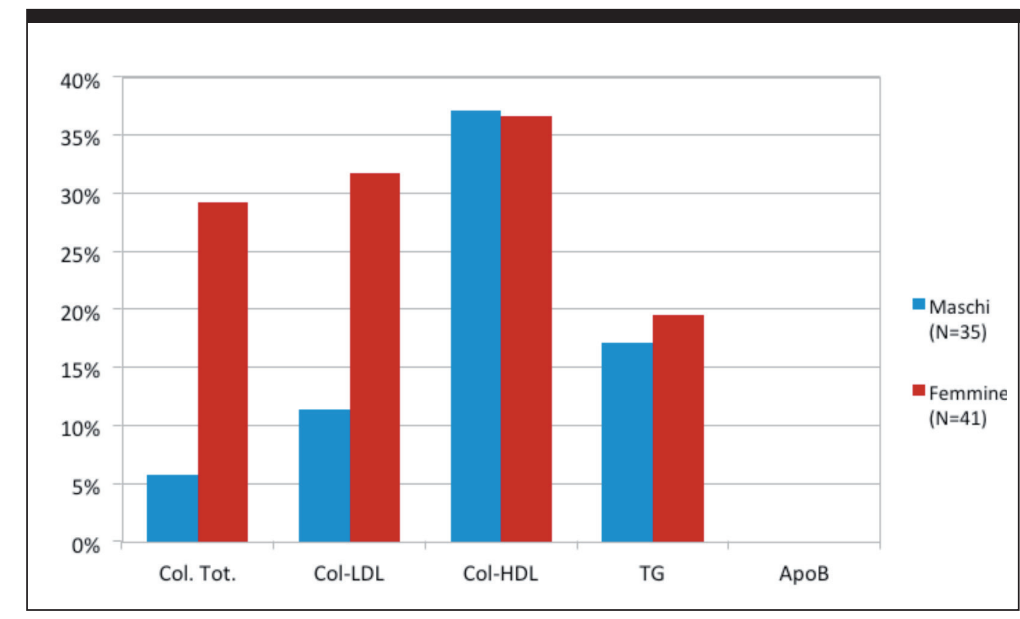

Figura 1. Frequenza di alterazione dei singoli parametri lipidici nella popolazione, divisa per sesso.

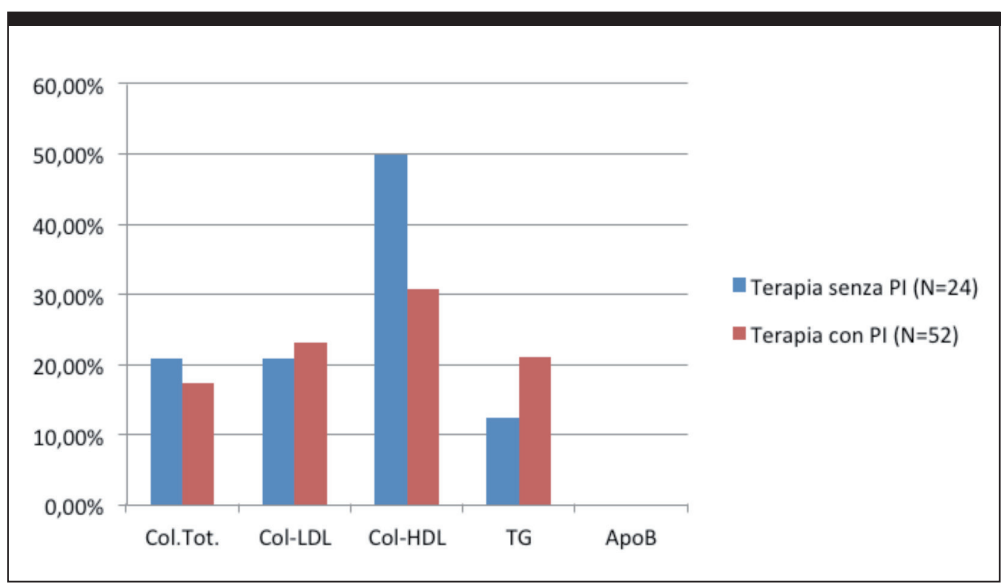

Figura 2. Frequenza di alterazione dei singoli parametri lipidici nella popolazione, divisa per tipo di regime terapeutico.

Dimostra inoltre che un profilo lipidico sfavorevole non sempre si associa alle manifestazioni "visibili" della lipodistrofia. Nella nostra popolazione è peraltro stata confermata una frequenza molto più elevata di lipodistrofia tra i pazienti che assumono PI (17.0\%) rispetto ai PI-free (2.6\%); il fatto che quest'ultima non sia zero, conferma infine il fatto che la lipodistrofia non è unicamente un effetto avverso della terapia.

L'infezione da HIV è in grado di modificare direttamente il metabolismo lipidico, agendo soprattutto sui livelli di HDL. Nel nostro studio, il Test di correlazione lineare di Pearson ha dato risultati coerenti con la letteratura, evidenziando una correlazione 
inversa debole tra la carica virale e il livello di HDL e una correlazione diretta debole tra la conta dei CD4 e il livello di HDL (con indici di Pearson rispettivamente pari a -0.019 e 0.086). I nostri dati confermano quindi, seppur con correlazioni molto deboli, quanto già rilevato negli adulti, ovvero che il livello di HDL è legato allo stato viro-immunologico. Anche per la correlazione tra livello di HDL e parametri infiammatori, la letteratura disponibile è limitata a studi svolti su soggetti adulti: nello studio SMART (8) era stata rilevata un'associazione inversa tra livelli di HDL e quelli di hs-PCR, IL-6 e D-dimero (parametri di infiammazione e coagulazione correlabili a patologia cardiovascolare). Nel nostro lavoro abbiamo considerato IL-6 e D-dimero, che hanno dato risultati diversi nel Test di correlazione di Pearson: tra IL-6 e HDL è stata evidenziata, come atteso, una correlazione inversa debole (indice di Pearson $=-0.073$ ), mentre per il D-dimero è risultata una correlazione diretta debole (indice di Pearson $=0.02$ ). Per questi due parametri, come anche per la carica virale, la maggior parte dei pazienti hanno valori non rilevabili perché inferiori ai limiti di laboratorio $(77.5 \%$ per IL-6, 91.6\% per D-dimero, $81.5 \%$ per la carica virale), che quindi vanno a posizionare la retta quasi orizzontalmente a livello dei limiti di laboratorio, giustificando almeno in parte la correlazione estremamente debole e in parte dubbia con le HDL. Nel complesso, quindi, la correlazione dei livelli di colesterolo HDL con i parametri relativi allo stato viro-immunologico e infiammatorio appare molto debole, talvolta di dubbio significato all'interno della nostra popolazione, rispetto a quanto riportato in letteratura nella popolazione adulta.

\section{Discussione}

Nei pazienti adulti con infezione da HIV, ed in parte anche nei pazienti pediatrici, l'aumentato rischio cardiovascolare è stato ormai dimostrato sia tramite misurazione di parametri laboratoristici, come quelli del profilo lipidico e quelli relativi allo stato infiammatorio, sia tramite esami strumentali, come la misurazione della Carotid Intima-Media Thickness e della Flow-Mediated Dilatation. Nella popolazione generale, disordini metabolici come I'insulino-resistenza e la dislipidemia sono associati ad un elevato rischio cardiovascolare (2); se questi disordini si presentano già in età pediatrica, il paziente è ad elevato rischio di presentarli anche nell'età adulta e di andare precocemente incontro ad aterosclerosi ed eventi cardiovascolari. In diversi studi su bambini e adolescenti con profilo lipidico alterato è stata rilevata la presenza di lesioni aterosclerotiche precoci $(14,15)$. Inoltre il rischio di patologia cardiovascolare è più elevato nei pazienti afro-americani rispetto ai caucasici e in bambini con familiarità per patologia cardiovascolare (16). La dislipidemia correlata alla CART è stata proposta come fattore determinante nella patogenesi della malattia cardiovascolare nei pazienti con infezione da HIV ed è stata descritta in coorti di bambini e adolescenti trattati con questo tipo di terapia, sebbene la prevalenza di questi disordini differisca significativamente tra gli studi $(10-12,17)$. L'uso di diverse combinazioni farmacologiche secondo le diverse linee guida nazionali e la diversa disponibilità di farmaci possono spiegare alcune di queste differenze; anche fattori socio-demografici, soprattutto quelli correlati alla dieta, possono essere in parte responsabili di queste divergenze nei risultati. Per quanto riguarda il nostro studio, abbiamo rilevato una quota elevata di pazienti con uno o più parametri lipidici alterati (58\%); tra i diversi parametri, quello più frequentemente alterato si è dimostrato essere il colesterolo-HDL, con livelli inferiori al valore desiderabile rilevati nel $36.8 \%$ dei pazienti. Suddividendo la popolazione secondo l'età ( $\leq 18$ anni e > 18 anni), abbiamo potuto evidenziare come per entrambi i gruppi il colesterolo-HDL fosse il parametro più frequentemente alterato, con una frequenza però nettamente maggiore tra i pazienti più giovani (53.3\%, contro il $26.1 \%$ degli adulti) e una differenza statisticamente significativa tra le due frequenze. Al contrario degli studi disponibili in letteratura $(11,12)$, nella nostra casistica non abbiamo evidenziato differenze significative nelle frequenze di alterazione dei diversi parametri lipidici tra il gruppo di pazienti che assume PI rispetto a quello che assume regimi terapeutici non contenenti questo tipo di farmaci. A dispetto dell'elevata prevalenza di dislipidemia, non è stato rilevato alcun valore glicemico indicativo di diabete o di alterata glicemia a digiuno, coerentemente con quanto riportato in letteratura .

In conclusione, alcuni dei fattori che vanno ad agire aumentando il rischio di aterosclerosi precoce e quindi di eventi cardiovascolari nella popolazione infetta da HIV (stato infiammatorio persistente ed effetti collaterali della CART) sono presenti in misura variabile in tutti i pazienti e non sono purtroppo 
al momento modificabili. Altri invece, come sovrappeso, inattività fisica, dislipidemia, insulino-resistenza/diabete, ipertensione ed abitudine al fumo, sono solo parzialmente legati alla malattia infettiva e al suo trattamento, dipendendo almeno in parte dalle abitudini di vita del paziente, e possono essere corretti con un miglioramento dello stile di vita o eventualmente con una terapia farmacologica. La prevenzione diventa quindi fondamentale, soprattutto parlando di popolazione pediatrica e giovane adulta; essa parte certamente dall'educazione ad una corretta alimentazione e ad uno stile di vita attivo, arrivando poi ad uno screening per la diagnosi precoce della patologia cardiovascolare. Sarebbe fondamentale a questo scopo anche la realizzazione di studi più approfonditi per poter stabilire linee guida che indichino, nei pazienti pediatrici, a quale età e con quali metodiche laboratoristiche e strumentali sia più adeguato iniziare una valutazione periodica del rischio cardiovascolare.

\section{BIBLIOGRAFIA}

1. Blanche S, Newell ML, Mayaux MJ, et al. Morbidity and mortality in European children vertically infected by HIV-1. The French Pediatric HIV Infection Study Group and European Collaborative Study. JAIDS 1997; 14: 442-50.

2. Rodger A, Lodwick R, Schechter M, et al. Mortality in well controlled HIV in the continuous antiretroviral therapy arms of the SMART and ESPRIT trials compared with the general population. AIDS 2013; 27: 973-9.

3. World-heart-federation.org. Cardiovascular disease risk factors | World Heart Federation. [online] Available at: http://www.worldheart-federation.org/press/fact-sheets/cardiovascular-disease-risk-factors/. Accessed 7 Jun. 2017.

4. Lang S, Mary-Krause M, Cotte L, et al. Increased risk of myocardial infarction in HIV-infected patients in France, relative to the general population. AIDS 2010; 24: 1228-30.

5. Non L, Escota G, Powderly W. HIV and its relationship to insulin resistance and lipid abnormalities. Translational Research 2017; 183: 41-56.

6. Da Cunha J, Morganti Ferreira Maselli L, Bassi Stern AC, Spada C, Bydlowski SP. Impact of antiretroviral therapy on lipid metabolism of human immunodeficiency virus-infected patients: Old and new drugs. World Journal of Virology 2015; 4: 56.

7. Palacios R, Santos J, Garcia A, et al. Impact of highly active antiretroviral therapy on blood pressure in HIV-infected patients. A prospective study in a cohort of naive patients. HIV Medicine 2006; 7: 10-5.

8. Duprez D, Kuller L, Tracy R, et al. Lipoprotein particle subclasses, cardiovascular disease and HIV infection. Atherosclerosis 2009; 207: 524-9.

9. Sherer Y, Shoenfeld Y. Mechanisms of Disease: atherosclerosis in autoimmune diseases. Nature Clinical Practice Rheumatology 2006; 2: 99-106.

10. Farley J, Gona P, Crain M, et al. Prevalence of Elevated Cholesterol and Associated Risk Factors Among Perinatally HIV-Infected Children (4-19 years old) in Pediatric AIDS Clinical Trials Group 219C. JAIDS 2005; 38: 480-7.

11. Carter R, Wiener J, Abrams E, et al. Dyslipidemia Among Perinatally HIV-Infected Children Enrolled in the PACTS-HOPE Cohort, 1999-2004. JAIDS 2006; 41: 453-60.

12. Lainka E, Oezbek S, Falck M, Ndagijimana J, Niehues T. Marked Dyslipidemia in Human Immunodeficiency Virus-Infected Children on Protease Inhibitor-Containing Antiretroviral Therapy. Pediatrics 2002; 110: e56.

13. Sculier D, Toutous-Trellu L, Verolet C, Matthes N, Lecompte T, Calmy A. Lipohypertrophy and metabolic disorders in HIV patients on antiretroviral therapy: a systematic multidisciplinary clinical approach. J Int AIDS Soc 2014; 17(4 Suppl 3): 19559.

14. Hanna, D. HIV Infection and Carotid Artery Intima-media Thickness: Pooled Analyses Across 5 Cohorts of the NHLBI HIV-CVD Collaborative. Clin Inf Dis 2016; 63: 249-56.

15. Charakida M, Donald A, Green H, et al. Early Structural and Functional Changes of the Vasculature in HIV-Infected Children: Impact of Disease and Antiretroviral Therapy. Circulation 2005; 112: 103-9.

16. Muntner P, Abdalla M, Correa A, Griswold M, Hall JE, Jones DW. Hypertension in Blacks: Unanswered Questions and Future Directions for the JHS (Jackson Heart Study). Hypertension 2017; 69: 761-9.

17. Blázquez D, Ramos-Amador JT, Saínz T, Mellado MJ, García-Ascaso M, De José MI. Lipid and glucose alterations in perinatallyacquired HIV-infected adolescents and young adults. BMC Infect Dis 2015; 15: 119. 